\title{
Term Abdominal Pregnancy with Live Baby: Case Report from Hiwot Fana Specialized University Hospital, Eastern Ethiopia
}

\author{
Tadesse Gure (D) \\ Semir Sultan' \\ Rehama Alishum' \\ Abdek Ali (iD) ${ }^{2}$ \\ Bayissa Dibaba' \\ Ibsa Usmael ${ }^{\prime}$ \\ Setegn Tsegaye' \\ 'Department of Gynecology and \\ Obstetrics, Haramaya University, Harar, \\ Ethiopia; ${ }^{2}$ Department of Gynecology \\ and Obstetrics, Jigjiga University, Jigjiga, \\ Ethiopia
}

\begin{abstract}
Purpose: To present a rare case of term abdominal pregnancy with live baby.
Observation: A 30-year-old gravida VI para V, all alive, woman who did not remember her last menstrual period but was stated amenorrheic for 9 months visited Hiwot Fana Specialized University Hospital (HFSUH) with abdominal pain of two weeks duration. The woman was diagnosed with a term abdominal pregnancy and underwent a laparotomy. The outcome of treatment was a 3200 gm healthy male baby and a total abdominal hysterectomy was done.
\end{abstract}

Conclusion and Importance: Term abdominal pregnancy is an extremely rare type of ectopic pregnancy that can cause diagnostic challenges and leads to life threatening conditions both before and after diagnosis because of the rupture of major blood vessels.

Keywords: abdominal pregnancy, ectopic, term, live baby, Ethiopia, Harar

\section{Introduction}

Abdominal pregnancy is one of the rare and life threatening types of ectopic pregnancy which has implanted in the peritoneal cavity outside of the uterus, fallopian tube and ovary. $^{1,2}$ It accounts for 1 per 10,000 births and 1.4\% of ectopic pregnancies. ${ }^{3,4}$ An abdominal pregnancy is the only type of ectopic pregnancy that can advance beyond 20 weeks of gestational age. ${ }^{5}$ It can be classified as primary and secondary. If the fertilization and implantation is in the peritoneal cavity from the outset, it is called a primary abdominal pregnancy, while if the abdominal pregnancy happened after tubal rupture or abortion or uterine rupture with secondary implantation over the peritoneum it is called a secondary type. ${ }^{2,5,6}$

The diagnosis of an abdominal pregnancy is made with a high index of suspicion since they have only vague, nonspecific symptoms and are often missed by imaging. ${ }^{2}$ Though, with ultrasound, there is a high possibility of missing an abdominal pregnancy, it is the first line diagnostic modality for abdominal pregnancy. ${ }^{3}$

Treatment of an abdominal pregnancy depends on gestational age, location of the implantation, placental attachment and hemodynamic stability of the patient.,

\section{Case Summary}

A 30-year-old gravida VI para-V, all delivered at home vaginally and alive. The last delivery was three years back. The women does not remember her last normal menstrual period for the current pregnancy, but stated to be amenorrheic for 9 
months, visited Hiwot Fana Specialized University Hospital (HFSUH) with the chief complaint of abdominal pain of two weeks duration. She never had antenatal care or an ultrasound examination for the index pregnancy. She came to the health facility for the first time since the conception of the current pregnancy.

She stated that she started to feel a dull aching abdominal pain all over the abdomen which later worsened and she started to feel shortness of breath. She also experienced increased nausea and vomiting and increased fetal movement compared to the previous five pregnancies. She reported to have had no vaginal bleeding.

On physical examination, she looks malnourished with prominent zygomatic bones. Her vital sign were blood pressure $120 / 80 \mathrm{mmhg}$, pulse rate 90 beats per minutes, temperature $36.9^{\circ} \mathrm{C}$ and respiratory rate 20 per minutes. All these records showed that the vital signs were within normal limits.
She has pink conjunctiva and non-icteric sclera. Her respiratory and cardiovascular examination is unremarkable. On abdominal examination, the pregnancy was a term sized, with easily palpable fetal parts, transverse lie, and positive fetal heartbeat of 152 beats per minute. There is no clear border of the uterus nor was there felt any signs of uterine contraction. On pelvic examination the cervix is closed, posterior and uneffaced.

After admission, the laboratory results showed that her hematocrit level was $33 \%$ and blood group was $\mathrm{O}$ positive. Ultrasound findings indicated intra-abdominal viable pregnancy with gestational age of 37 weeks and 5 days, transverse fetal lie, empty uterus, fluid inside the cul-de-sac and Doppler evaluation showed vascularization of uterine fundus (Figure 1).

Based on the above findings, she was diagnosed to have a term intra-abdominal pregnancy and emergency laparotomy

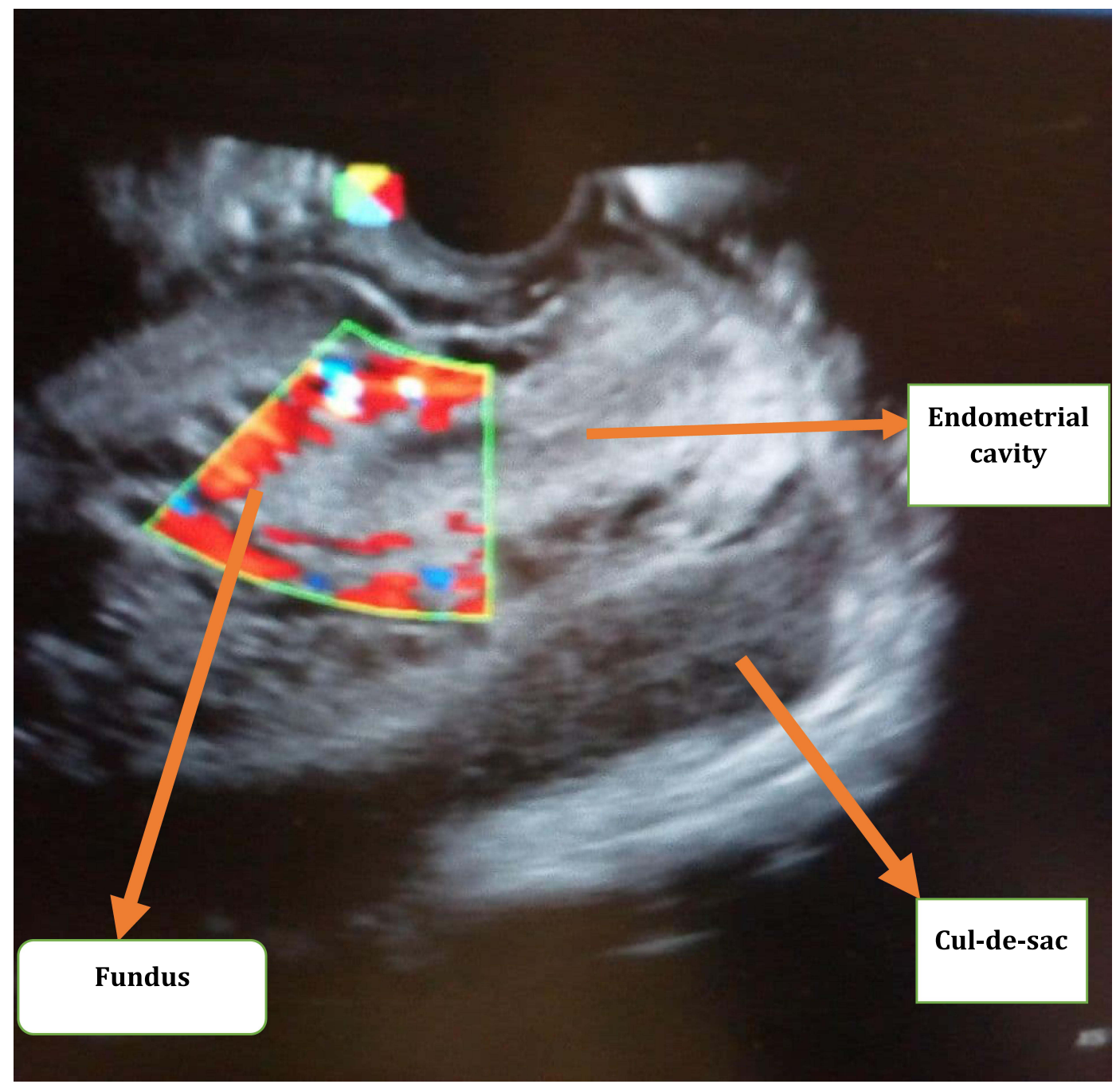

Figure I Ultrasound picture showing empty uterus with fundal vascularization. 
was decided. Three units of blood was prepared as an emergency in case excessive bleeding happened. The women was counselled on her condition and the possible options of intraoperative management and complications. Then, after written consent was taken, prophylactic antibiotics were given and the patient taken to the operation theater. Under general anesthesia she underwent a laparotomy. The findings were an intraabdominal pregnancy with intact gestational sac. The placenta was attached to the uterine fundus and the omentum. The uterus looked normal grossly except the fundal part where the placenta covered it. Both tubes and ovaries also looked healthy.

\section{Operative Procedure}

the abdomen was entered through infra-umbilical mid line incision. While peritoneum was opened the membrane ruptured and meconium stained amniotic fluid leaked and the umbilical cord prolapsed (Figure 2). Then the peritoneum and membrane were bluntly dissected to deliver a $3200 \mathrm{gm}$ male alive baby with APGAR of 7 and 8 at first and fifth minutes, respectively (Figure 3). The placenta was attached to the omentum and fundus of the uterus getting blood supply from both (Figure 4). For this clamping, cutting and transfixing of the omentum attached to the placenta was done. After the placenta was separated from the omentum a total abdominal hysterectomy was done in orderly fashion without removing the placenta from the uterus (Figure 5). The placenta and the uterus were removed together, hemostasis secured, abdomen cleaned and closed in layers. Patient was extubated and transferred to a recovery room with stable vital signs. The total estimated blood loss was $1200 \mathrm{~mL}$ and postoperatively she was transfused with two units of crossmatched blood and given therapeutic antibiotics. After eight days of post-operative stay in the hospital, the mother and the baby were discharged healthy.

\section{Discussion}

Ectopic pregnancy is a type of pregnancy and is defined as implantation of the blastocyst outside of the endometrial lining. These implantations can occur inside the tube (tubal pregnancy), on the ovary (ovarian pregnancy), inside the abdomen (abdominal pregnancy), inside the cervix (cervical pregnancy), inside the cesarean scar, ... etc. The prevalence of ectopic pregnancy is $1-2 \%$. Among all types of ectopic pregnancy $95-98 \%$ occur inside the uterine tubes. ${ }^{5,7,8}$

Abdominal pregnancy is an extremely rare but life threatening type of pregnancy which is implanted in the peritoneal cavity outside of the uterine cavity and fallopian

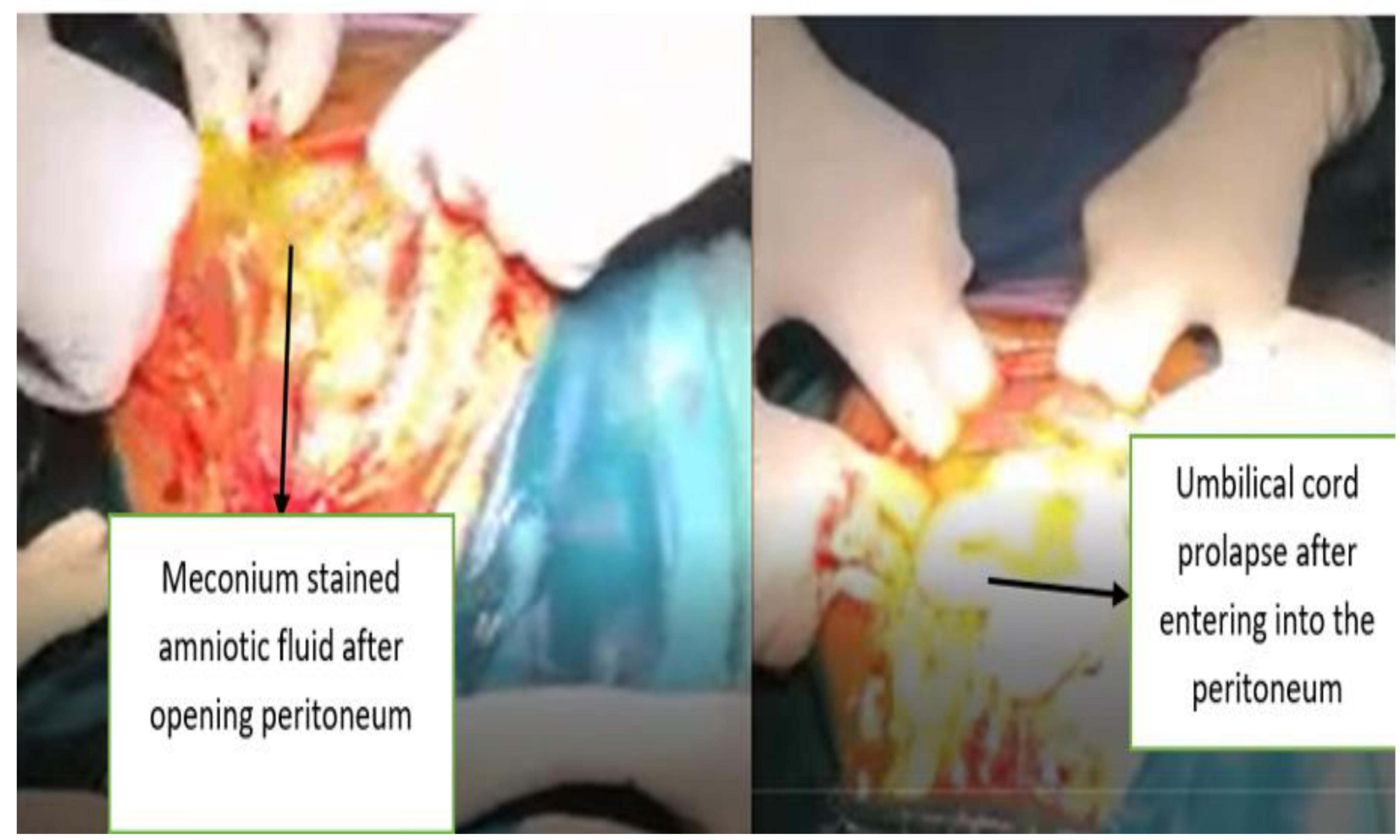

Figure 2 Amniotic fluid leaking and cord prolapsing upon abdominal entry. 


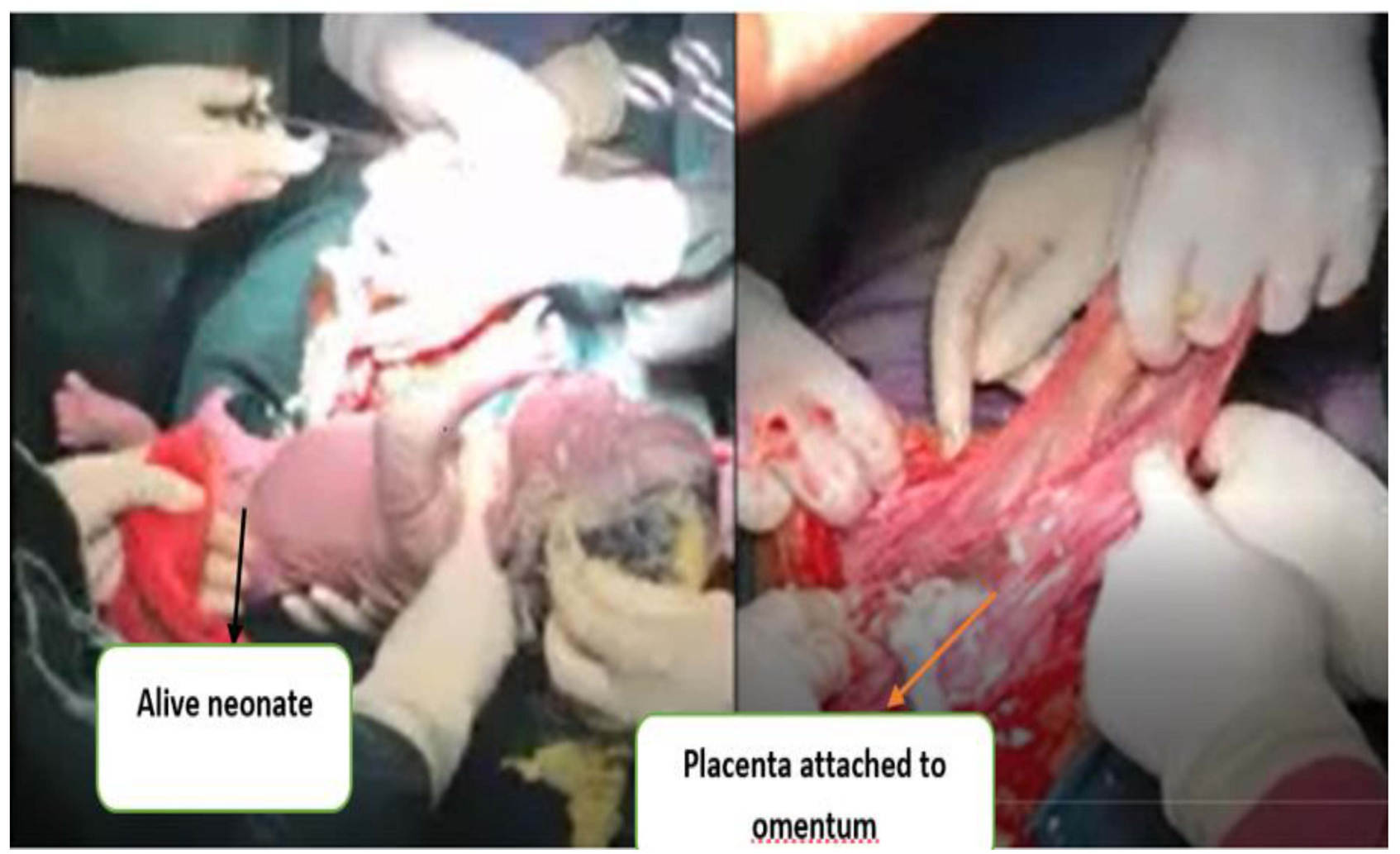

Figure 3 Live baby and placenta attachment to the omentum.

tube. ${ }^{1,2}$ It accounts for 1 per 10,000 births and accounts for $1.4 \%$ of ectopic pregnancies. Abdominal pregnancy is usually misdiagnosed as an intrauterine pregnancy during antenatal care. ${ }^{2}$ In the first trimester, it is missed as tubal pregnancy and in late trimester it is missed as intrauterine pregnancy. ${ }^{1,9-11}$ Abdominal pregnancies can be classified into primary and secondary type based on the primary site of the gestational implantation. The primary type of abdominal pregnancy is a type of ectopic pregnancy in which the blastocyst primary implanted inside the peritoneal cavity while the secondary type of abdominal pregnancy is initially implanted either in the tube, uterus or ovary but later migrates out due to tubal or uterine rupture or tubal abortion, then it will be implanted into the peritoneal cavity. ${ }^{1,10}$ In 1942, Studdiford introduced the following three diagnostic criteria for primary abdominal pregnancies. These are; 1 . both tubes and ovaries must be in a normal condition with no evidence of recent or remote injury, 2. no evidence of utero-peritoneal fistula should be found; and 3. the pregnancy must be related exclusively to the peritoneal surface and be early enough. ${ }^{12}$ In this case though the bilateral tube and ovary looked normal, the placenta is attached and getting blood supply from both the omentum and fundus of the uterus. For this reason this patient can be diagnosed as the secondary type of abdominal pregnancy.

Though the exact etiology of primary abdominal pregnancy is unknown there are different risk factors identified. These include tubal damage, pelvic inflammatory diseases, multi-parity, in vitro fertilization, and others. In the current patient, except multi-parity, there were no identified risk factors. ${ }^{1}$

Diagnosis of extra uterine pregnancy is not easy and a high index of suspicion is very important to diagnose ectopic pregnancy. ${ }^{2,10,13}$ Based on the clinical feature of amenorrhea, abdominal pain and vaginal bleeding one can suspect ectopic pregnancy. ${ }^{10}$ Tubal pregnancy, which is the commonest type of ectopic pregnancy, usually rupture or abort in early trimester and are diagnosed early. However, abdominal pregnancy is the only ectopic pregnancy that can advance and reach term. In this patient, she did not go for an ANC for the current pregnancy and it continued to term.

To reach a diagnosis, in addition to clinical suspicion, imaging, especially ultrasound, is very important. The ultrasound features of abdominal pregnancy include: 


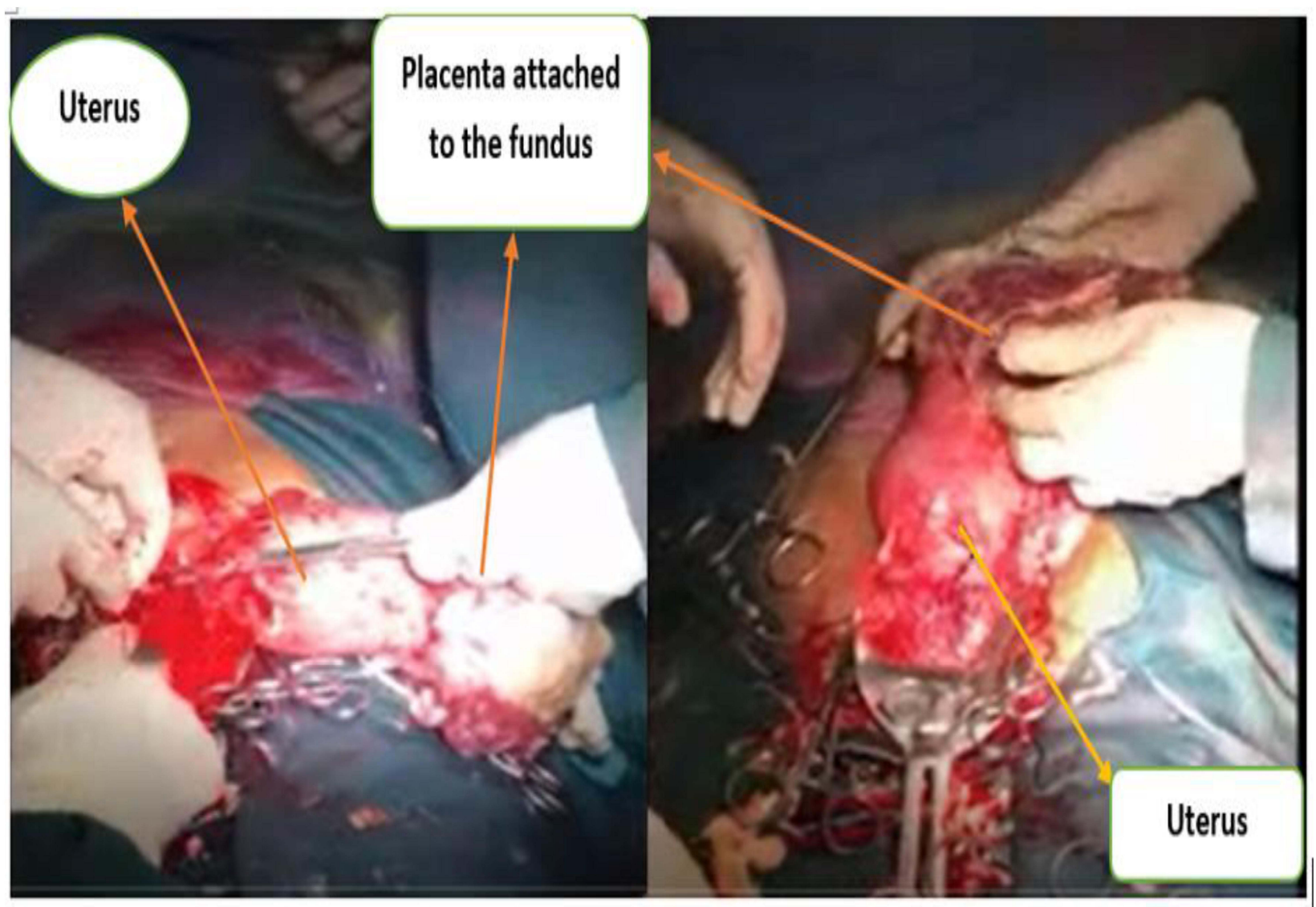

Figure 4 Picture showing placental attachment to fundus of the uterus.

A. An absence of myometrial tissue between the maternal bladder and the pregnancy, abdominal wall and pregnancy. B. An empty uterus. C. Poor definition of the placenta. D. Oligohydramnios. E. Unusual fetal lie. ${ }^{1,14}$ In our case the ultrasound findings are similar to the ones indicated above. Rarely magnetic resonance imaging (MRI) can be used for the diagnosis of abdominal pregnancy. ${ }^{10,15}$

Abdominal pregnancy is associated with life threatening complications both to the mother and fetus. It may lead to fetal and maternal death if there is intra peritoneal bleeding due to vascular rupture. ${ }^{13,16}$ Once diagnosis is made treatment depends on gestational age, hemodynamic stability and site of implantation inside the peritoneal cavity. ${ }^{17}$ In early trimester laparoscopic management, vascular embolization and feticidal are possible management options. In a late trimester laparotomy is the main stay of management. ${ }^{1}$ The fetus can be delivered easily but the decision about the management of the placenta should be made cautiously since removal of the placenta may cause torrential bleeding and maternal death. ${ }^{1,13,18}$ Removal of placenta should only be tried if attachment is simple and easy to remove. ${ }^{10,17}$ In this woman, the placenta was attached to the omentum which was easily separated and it was also attached to the fundus of the uterus so a hysterectomy was done en bloc with placenta. If the placenta is attached to major vessels or pelvic side wall it is recommended to leave the placenta in situ and give methotrexate. If the placenta is left in situ the placenta may become necrotic and super infected increasing the risk of abscess formation and sepsis so post-operative follow up is very important. ${ }^{1,19}$

\section{Conclusion}

Term abdominal pregnancy is a rare but life-threatening ectopic pregnancy that needs a high index of suspicion for diagnosis, a good plan and adequate preparation for surgery is very important to prevent maternal and fetal death. 


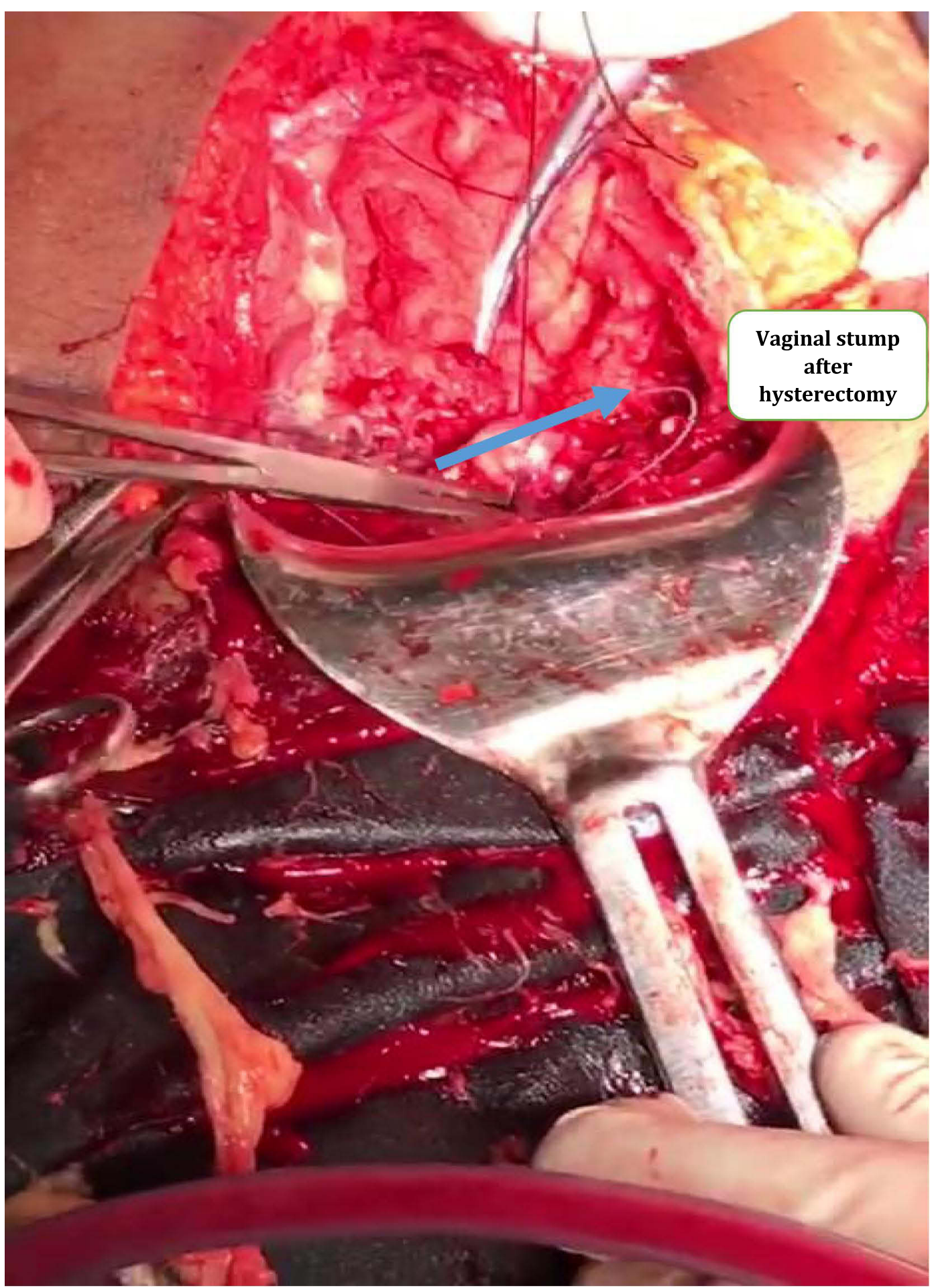

Figure 5 Figure showing vaginal stump after hysterectomy.

\section{Patient Consent}

Informed written consent has been obtained from the patient for all case details and images published. No institutional approval was required for the publishing of this case report.

\section{Acknowledgments}

I would like to thank Dr. Nega Assefa and Dr. Abera

Kenay for editing and commenting on this case report. 


\section{Author Contributions}

All authors are involved in diagnosing and treatment including operation, whilst the corresponding author wrote the case report. All authors contributed to data analysis, drafting or revising the article, gave final approval of the version to be published, agreed to the journal submitted to, and agree to be accountable for all aspects of the work.

\section{Funding}

No grant support or funding was given.

\section{Disclosure}

The authors have no financial or other conflicts of interest for this work.

\section{References}

1. Huang K, Song L, Wang L, Gao Z, Meng Y, Lu Y. Advanced abdominal pregnancy: an increasingly challenging clinical concern for obstetricians. Int J Clin Exp Pathol. 2014;7:5461.

2. Mengistu Z, Getachew A, Adefris M. Term abdominal pregnancy: a case report. J Med Case Rep. 2015;9(1):168. doi:10.1186/s13256015-0635-3

3. Adesiyun AG, Audu AI. Term extrauterine pregnancy in a Nigerian mother: a complication of uterine dehiscence. Arch Gynecol Obstet. 2009;279(1):75-77. doi:10.1007/s00404-008-0641-4

4. Igwemadu G, Tunde-Olatunji O, Akunaeziri U, Enang E. Advanced abdominal pregnancy: the challenges of management. Niger $J$ Med. 2020;29(3):514. doi:10.4103/NJM.NJM 3420

5. Masukume G. Live births resulting from advanced abdominal extrauterine pregnancy, a review of cases reported from 2008 to 2013. Wedmed Cent Obstet Gynecol. 2014;5(1):2-13.

6. Rafal W, Annabel L, Jochen M. Primary omental pregnancy with secondary implantation into posterior cul-de-sac: laparoscopic treatment using hemostatic matrix. J Minim Invasive Gynecol. 2015;22 (3):501-503. doi:10.1016/j.jmig.2014.06.008
7. Singh M, Singh R, Singh AB. A rare location of a repeat ectopic pregnancy: a case report. Cureus. 2021. doi:10.7759/cureus.15982

8. Brady PC. New evidence to guide ectopic pregnancy diagnosis and management. C Rev Artic. 2017;72:618-625.

9. Nassali MN, Benti TM, Bandani-Ntsabele M, Musinguzi E. A case report of an asymptomatic late term abdominal pregnancy with a live birth at 41 weeks of gestation. BMC Res Notes. 2016;9(1). doi:10.1186/s13104-016-1844-6

10. Pednekar G, Jindal D, Surve S. Successful outcome in a near term secondary abdominal pregnancy presenting as diagnostic dilemma. IOSR J Med Dent Sci. 2021;8:83-86.

11. Arga M, NaliniSharma VJ. Case report: case report. Can Fam Physician. 2001;47(10):788-789.

12. Weiner JJ. Primary abdominal pregnancy. Am J Surg. 1944;65 (2):288-289. doi:10.1016/S0002-9610(44)90334-X

13. Dahab AA, Aburass R, Shawkat W, Babgi R, Essa O, Mujallid RH. Full-term extrauterine abdominal pregnancy: a case report. $J$ Med Case Rep. 2011;5(1). doi: 10.1186/1752-1947-5-531

14. Abdul Jabbar NAR, Saquib S, Talha WEM. Successful management of abdominal pregnancy: two case reports. Oman Med J. 2018;33 (2):171-175. doi:10.5001/omj.2018.32

15. Lockhat F, Corr P, Ramphal S, Moodley J. The value of magnetic resonance imaging in the diagnosis and management of extra-uterine abdominal pregnancy. Clin Radiol. 2006;61(3):264-269. doi:10.1016/j.crad.2005.10.013

16. Poddar DL. Abdominal pregnancy: Report of ten cases with analysis. J Obstet Gynaecol India. 1956:16-25. Available from: http://www. jogi.co.in/articles/view.php?id=MjQw. Accessed September 27, 2021.

17. Kim MJ, Bae JY, Seong WJ, Lee YS. Sonographic diagnosis of a viable abdominal pregnancy with planned delivery after fetal lung maturation. J Clin Ultrasound. 2013;41(9):563-565. doi:10.1002/ jcu. 22010

18. Reshma G, Edward P, Robert G. Abdominal ectopic pregnancy. Baylor Univ Med Cent Proc. 2021;34(4):530-531. doi:10.1080/ 08998280.2021 .1884932

19. Fessehaye A, Gashawbeza B, Daba M, Arusi M, Terefe T. Abdominal ectopic pregnancy complicated with a large bowel injury: a case report. J Med Case Rep. 2021;15(1):1-5. doi:10.1186/s13256-02102713-9

\section{Publish your work in this journal}

The International Medical Case Reports Journal is an international, peer-reviewed open-access journal publishing original case reports from all medical specialties. Previously unpublished medical posters are also accepted relating to any area of clinical or preclinical science. Submissions should not normally exceed 2,000 words or 4 published pages including figures, diagrams and references. The manuscript management system is completely online and includes a very quick and fair peer-review system, which is all easy to use. Visit http://www.dovepress.com/testimonials.php to read real quotes from published authors. 